\title{
Lateral-Torsional Buckling of beams with warping restraints at Supports
}

\author{
Maxime Lebastard ${ }^{1,2}$, Maël Couchaux ${ }^{2}$, Alain Bureau ${ }^{1}$, M ohammed Hjiaj² $^{2}$
}

\author{
${ }^{1}$ Centre Technique Industriel de la Construction Métallique \\ Espace technologique - L'Orme des Merisiers Immeuble Apollo \\ 91193 Saint-Aubin, France \\ ${ }^{2}$ Institut National des Sciences Appliquées \\ 20 Avenue des Buttes de Coësmes \\ 35708 Rennes, France \\ Correspondence Maxime LEBASTARD Research Engineer \\ Email: mlebastard@cticm.com
}

\begin{abstract}
In practice, the elastic lateral-torsional buckling of doubly symmetric I-section steel members is analyzed assuming free warping at supports. However, welded stiffeners, bolted end-plate con-nections or column base plates maintain elastically the opposite flange out-of-plane rotations and thus induce substantial warping restraints.

This paper proposes analytical formulations for the evaluation of the elastic critical bending mo-ment for lateral-torsional buckling of beams taking into account warping restraints at supports. Assuming series of displacement and rotation fields containing one or two terms, the energy method permits to derive expressions of the critical bending moment under constant and linear distribution of the bending moment. A single expression of the warping coefficient $k_{w}$ is proposed whatever the shape of the bending moment diagram. The factor $C_{1}$ can be conveniently calculated by multiplying two coefficients that depend on the shape of the bending moment diagram and on the warping restraint stiffness, respectively.

Finite Elements analyses of beams with warping restraints at supports have been performed con-sidering a beam element model developed with LTBeamN and a shell element model created in ANSYS. The analytical model is in good agreement with the numerical results obtained by the two finite element models.
\end{abstract}

Keywords Elastic critical bending moment, Warping restraints, Lateral-torsional buckling.

1

Introduction

The elastic critical bending moment is commonly determined considering theoretical fork support conditions at both ends, i.e. fixed lateral displacement and torsional rotation but free warping. Because very few authors have proposed expressions for the critical moment of a beam elastically restraint against warping, the influence of the warping restraints on the critical moment is usually neglected.

In this paper, the influence of warping restraints on the criticalbending moment is investigated. After a brief review of the literature about the determination of the elastic critical moment of a uniform beam with doubly symmetric cross-section and with warping restraints at supports (see Figure 1), an analytical model is described. Considering appropriate series of displacement and rotation fields, the energy method is used to derive a formula of the elastic critical bending moment under constant and linear distribution of the bending moment. It is shown that the use of displacement and rotation fields approximated with one term is correct for uniform bending moment but for a linear moment diagram two terms are necessary.

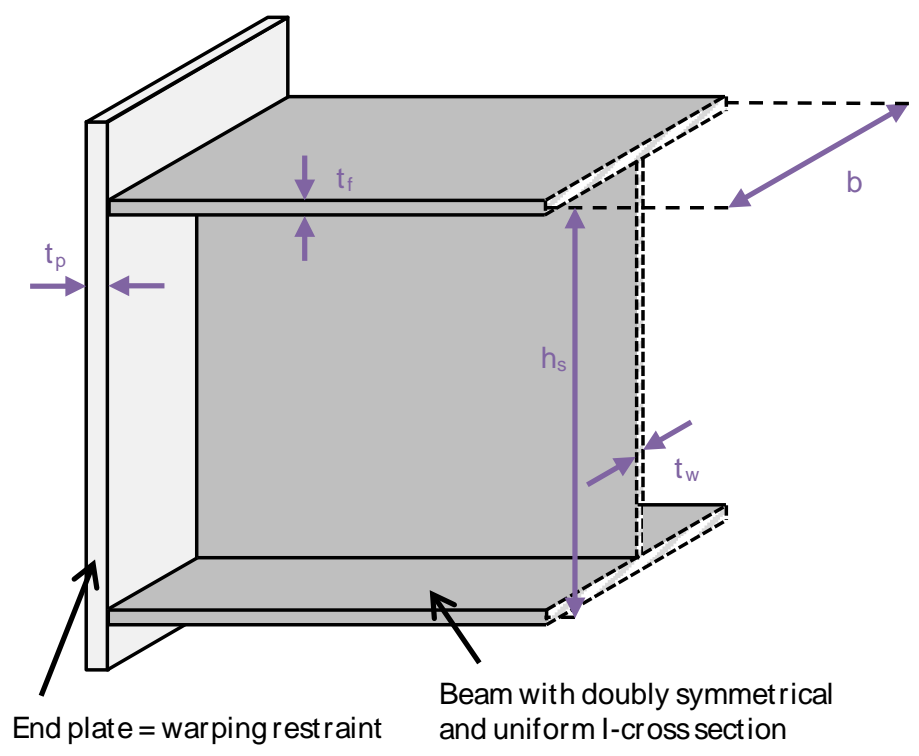

Figure 1 End of a beam with plat es restraining warping

The derived expression for the critical bending moment depends on 
the warping stiffness through the coefficients $C_{1}$ and $k_{\mathrm{w}}$. While the warping coefficient $k_{\mathrm{w}}$ has a single expression, $C_{1}$ also depends on the shape of the bending moment distribution.

Finally, the predictions of the analytical model are compared against finite element results of beams with warping restraints at supports, computed either using beam elements model with the software LTBeamN [1] or shell elements model with ANSYS. A good agreement between the analytical model and finite element analyses is observed.

\section{$2 \quad$ Literature overview}

In the French National Annex to Eurocode 3 Part 1-1 [2],the formula of the elastic critical bending moment can account for warping restraints only through the warping coefficient $k_{\mathrm{w}}$. No expression is given to determine $k_{w}$ considering potential warping restraints. The critical moment of a beam with doubly symmetric cross-section and with free out-of-plane rotation considering a linear distribution of the bending moment is:

$$
M_{c r}=C_{1} \frac{\pi^{2} E I_{\mathrm{z}}}{L^{2}}\left\{\sqrt{\left(\frac{1}{k_{\mathrm{w}}}\right)^{2} \frac{I_{w}}{I_{\mathrm{z}}}+\frac{G I_{\mathrm{t}} L^{2}}{E I_{\mathrm{z}} \pi^{2}}}\right\}
$$

Where:

- $C_{1}$ : Equivalent uniform moment factor

- $E$ : Modulus of elasticity

- $\quad I_{z}:$ z-axis second moment of area (see Figure 2)

- $\quad L$ : distance between lateral restraints

- $\quad I_{w}:$ Warping constant

- $\iota_{\mathrm{t}}$ : Torsional constant

- $\quad G$ : Shear modulus.

The warping coefficient $k_{w}$ varies between 0,5 when warping is fixed to 1 when warping is free. However, no expression is given to compute $k_{\mathrm{w}}$ and it is assumed that $C_{1}$ depends only on the bending moment diagram without any influence of the warping restraints.
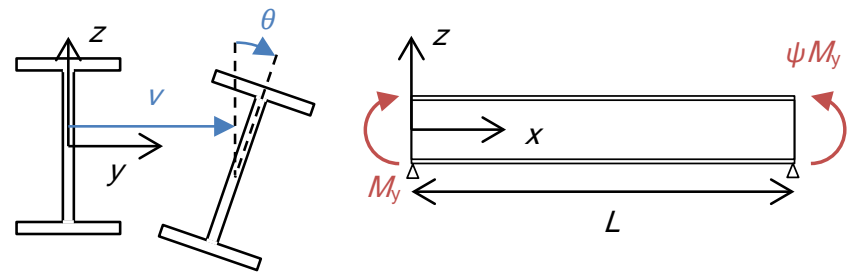

Figure 2 Axes and notations for the analytical derivation

Based on the work of Lindner and Gietzelt [3], it has been suggested in [4] to evaluate $k_{w}$ for I-beams with end plates at both ends using the following expression:

$$
\begin{gathered}
k_{w}=1-\frac{0.5}{1+\frac{2 E I_{w}}{c_{w} L}} \\
c_{w}=\frac{1}{3} G b t_{p}^{3} h_{s}
\end{gathered}
$$

Where:

- $\quad c_{w}$ : Stiffness of the warping restraints (see Figure 3)

- $\quad t_{\mathrm{p}}$ : Thickness of the end plates

- $h_{\mathrm{s}}$ : Distance between flanges centres.

Therefore, the warping coefficient depends on the warping stiffness
$E_{w}$, the length $L$ of the beam, and on the stiffness of the warping restraint $c_{\mathrm{v}}$. The bending moment distribution assumed for expression (2) is not specified. No expression is given for $C_{1}$; the values given in the French National Annex [2] considering free warping at supports should ther efore be used.

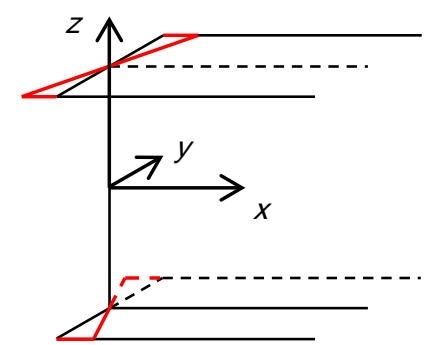

a) Free warping

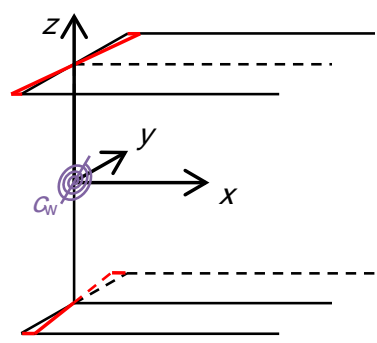

b) Warping restraint
Figure 3 Flanges longitudinal displacements when warping is a) free and b) restrained

For a uniform bending moment distribution, Pi and Trahair [5] proposed the following approximation for $k_{\mathrm{w}}$ :

$$
k_{w}=\frac{6,5+\frac{c_{w} L}{E I_{w}}}{6,5+2,25 \frac{c_{w} L}{E I_{w}}}
$$

Details on the derivation of expressions (2) to (4) in references [3][5] are missing. Piotrowski and Szychowski [6] used the energy method to propose a general expression of the elastic critical moment accounting for warping restraints. The out-of-plane displacement vand torsional rotation $\theta$ are approximated using power polynomials. The proposed expression for the critical moment of a beam under linear bending moment with free out of plane rotation is:

$$
M_{c r}=D_{1} \frac{\sqrt{E I_{z}\left(C_{1} G I_{T} L^{2}+C_{2} E I_{\mathrm{w}}\right)}}{C_{3} L^{2}}
$$

The values of $C_{1}, C_{2}, C_{3}$ and $D_{1}$, depending on the ratio $\psi$ between end moments, are given in Table 1.

Table 1 Coefficients to det ermine the critical moment

\section{$-0,5<\psi \leq-1$}

$$
\begin{array}{ll}
C_{1} & 57,6\left(1,457-2,4 \kappa_{\mathrm{w}}+\kappa_{\mathrm{w}}^{2}\right) \\
C_{2} & 691,2\left(1,2-\kappa_{\mathrm{w}}\right) \\
C_{3} & \sqrt{(1+\psi)^{2}\left(2,124-3,497 \kappa_{\mathrm{w}}+1,440 \kappa_{\mathrm{w}}^{2}\right)} \\
D_{1} & \begin{array}{l}
1,018+0,000297 \kappa_{\mathrm{w}}^{2}-0,009 \kappa_{\mathrm{w}}^{6}-e^{-2,5 \psi}\left(0,108-0,001 \kappa_{\mathrm{w}}-\right. \\
\left.0,014 \kappa_{\mathrm{w}}^{6}\right)
\end{array} \\
\hline & \multicolumn{1}{c}{-\mathbf{1} \leq \mathbf{\psi} \leq \mathbf{0 , 5}} \\
\hline C_{1} & 345,75\left(1,457-2,4 \kappa_{\mathrm{w}}+\kappa_{\mathrm{w}}^{2}\right) \\
C_{2} & 4148,98\left(1,2-\kappa_{\mathrm{w}}\right) \\
C_{3} & \sqrt{(1-\psi)^{2}\left(1,681-2,880 \kappa_{\mathrm{w}}+1,235 \kappa_{\mathrm{w}}^{2}\right)} \\
D_{1} & 1,068+0,014 \kappa_{\mathrm{w}}^{2}-e^{3 \psi}\left(1,704+0,099 \kappa_{\mathrm{w}}+0,215 \kappa_{\mathrm{w}}^{6}\right)
\end{array}
$$


The index of fixity against warping $\kappa_{\mathrm{w}}$ varies between 0 when warping is free, and 1 when warping is fixed. It can be obtained by the following expression:

$$
\kappa_{w}=\frac{1}{1+\frac{2 E I_{w}}{c_{w} L}}
$$

From expression (5), the warping coefficient is identified as :

$$
k_{w}=\frac{\pi}{2 \sqrt{3}} \sqrt{\frac{1,457-2,4 \kappa_{w}+\kappa_{w}^{2}}{1,2-\kappa_{w}}}
$$

The coefficient $C_{1}$ (when referring to equation (1)) is extracted from expression (5) and depends on the bending moment distribution, through the ratio $\psi$, and the warping restraints, through $\kappa_{\mathrm{w}}$. Some values of $C_{1}$ are given in Table 2 according to expression (5) and according to TableM 1 from the French National Annex to Eurocode 3 Part 1-1 [2].

Table 2 highlights the evolution of the coefficient $C_{1}$ with bending moment distribution and warping restraints. The values of $C_{1}$ when warping isfully restrained increase from $10 \%$ when the bending moment is constant to more than $30 \%$ when $\psi$ is equal to -1 when compared to free warping.

Besides, the values of $C_{1}$ given by the French National Annex [2] are lower than those given by Piotrowski [6] for free warping. Finite element analyses should be performed to assess the safety level of the approaches of the French National Annex and of Piotrowski.

Table $2 C_{1}$ for different values of $\psi$

\begin{tabular}{ccccccc}
\hline Reference & $\boldsymbol{\kappa}_{\mathrm{w}}$ & $\mathbf{1}$ & $\mathbf{0 , 5}$ & $\mathbf{0}$ & $\mathbf{- 0 , 5}$ & $\mathbf{- 1}$ \\
\hline $\begin{array}{c}\text { French NA } \\
\text { [2] }\end{array}$ & 0 & 1,00 & 1,31 & 1,77 & 2,33 & 2,55 \\
$\begin{array}{c}\text { Piotrowski } \\
\text { [6] }\end{array}$ & 0 & 1,010 & 1,317 & 1,822 & 2,527 & 2,709 \\
& $\infty$ & 1,114 & 1,458 & 2,040 & 3,010 & 3,566 \\
\hline
\end{tabular}

Simple expressions have been proposed to take into account the effect of warping restraints on the elastic critical moment, mainly through the warping coefficient $k_{\mathrm{w}}$. However, expressions (2) and (4) seem to be valid only for beams under uniform bending moment. Piotrowski [6] proposed to account for the bending moment distribution but the expressions are complex for a daily use in design office. Expressions based on consistent derivation of an analytical model are yet to be developed.

\section{Analytical Model}

\subsection{Introduction}

The energy method is used to derive an analytical expression of the elastic critical bending moment of a beam with warping restraints at supports. Firstly, the model is defined in the simple case of a beam under uniform bending moment in §3.2, using one term for the outof-plane displacement $v$ and the torsional rotation $\theta$ fields. A second term is then added to enhance the displacement and rotation fields in presence of a linear distribution of the bending moment in §3.3.
- Uniform beam with doubly symmetrical I-cross-section

- Fork supports conditions

- Warping restraints at supports (stiffness $C_{W}$ ).

Figure 2 present the coordinate system, consistent with Eurocode 3 Part1-1 [7] and notations used to develop the analytical model.

\subsection{Uniform bending moment distribution}

\subsubsection{Displacementsand rotations fields}

For a beam under constant bending moment $M_{\mathrm{y}, 0}$ with free warping and out-of-plane rotation at both supports, the exact solution of the lateral displacement and the torsional rotation has the shape of half of a sine-wave:

$$
\begin{aligned}
& v(x)=v_{0} \sin \left(\frac{\pi x}{L}\right) \\
& \theta(x)=\theta_{0} \sin \left(\frac{\pi x}{L}\right)
\end{aligned}
$$

Where:

\section{- $\quad v_{0}$ : Magnitude of the lateral displacement}

- $\theta_{0}$ : Magnitude of the torsional rotation.

However, for fixed warping at supports, obtaining the exact expression of the torsional rotation is not obvious. Vlasov [8] and Djalaly [9] have proposed the following approximation, considering a cosine-wave:

$$
\theta(x)=\theta_{0}\left[1-\cos \left(2 \frac{\pi x}{L}\right)\right]
$$

Elastic warping restraints at beam ends induce the following boundary conditions between the bimoment $B$, the warping stiffness $C_{w}$, and the torsional rotation:

$$
B(0)=-c_{\mathrm{w}} \frac{d \theta}{d x}(0) \quad \text { and } \quad B(L)=c_{\mathrm{w}} \frac{d \theta}{d x}(L)
$$

Besides, the bimoment is connected to the beam's warping stiffness and torsional rotation by:

$$
B(x)=-E I_{\mathrm{w}} \frac{d^{2} \theta}{d x^{2}}(x)
$$

The rotation has to comply with the following kinematic boundary conditions at supports, obtained by combining expressions (11) and (12):

$$
\frac{d^{2} \theta}{d x^{2}}(0)=\frac{c_{\mathrm{w}}}{E I_{\mathrm{w}}} \frac{d \theta}{d x}(0) \quad \text { and } \quad \frac{d^{2} \theta}{d x^{2}}(L)=-\frac{c_{\mathrm{w}}}{E I_{\mathrm{w}}} \frac{d \theta}{d x}(L)
$$

An approximate distribution of the torsional rotation is developed from equations (9) and (10), in agreement with boundary conditions (13):

$$
\theta(x)=\theta_{0}\left\{4 \pi \sin \left(\frac{\pi x}{L}\right)+\frac{c_{\mathrm{W}} L}{E I_{\mathrm{w}}}\left[1-\cos \left(2 \frac{\pi x}{L}\right)\right]\right\}
$$

The torsional rotation distribution and its first derivative are compared to finite element analyses. Figure 4 shows the results given by equation (14) and the results of an analysis performed with LTBeamN [1] using beam elements. The example correspond to a 8 $\mathrm{m}$-span beam under uniform bending moment with a $800 \times 8$ web, 200x14 flanges and end-plates of $30 \mathrm{~mm}$ thickness.

The analytical model relies on the following assumptions: 


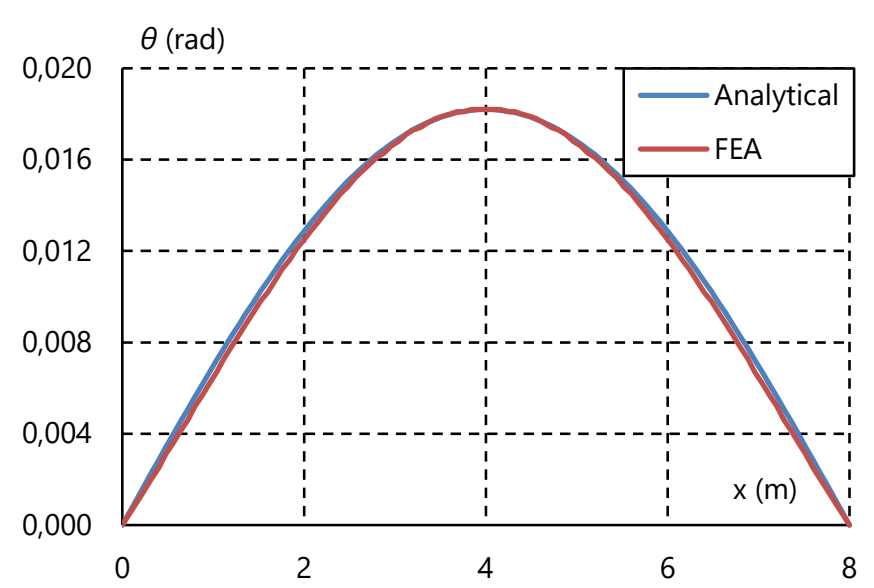

a) $\theta(x)$ $\theta^{\prime}(\mathrm{rad} / \mathrm{m})$

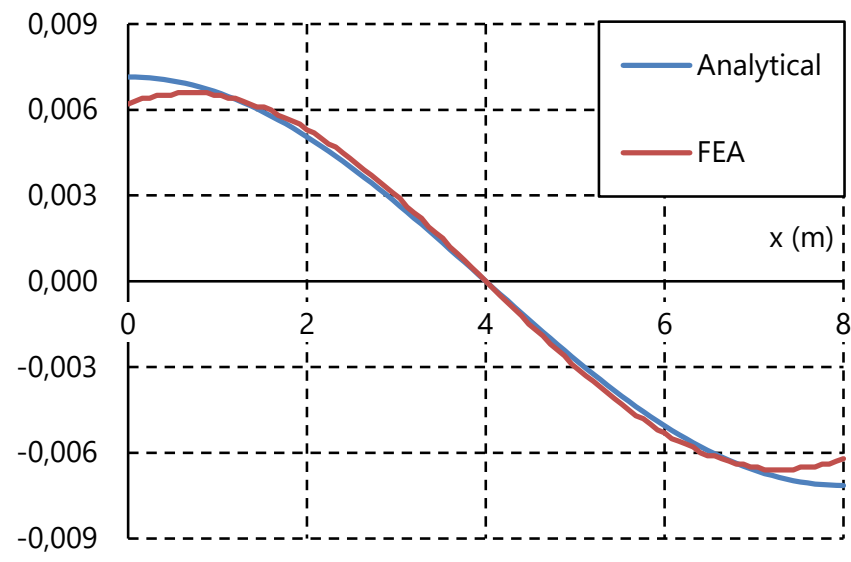

b) $\theta^{\prime}(x)$

Figure 4 a) Rotation and b) first derivative along a beam with warping restraints under const ant bending moment

The analytical and finite element models give very close results. Some discrepancies appear when looking at the derivative at supports, explaining that small differences might be encountered between the analytical model to be developed and the numerical model.

\subsubsection{Potential energy}

The energy method is based on the minimisation of the potential energy $\Pi$, defined as the difference between the elastic strain energy $U$ and the work of external loads $W$ that can be expressed as (see Timoshenko [10]):

$$
\delta^{2} \Pi=\delta^{2}(U-W)=0
$$

The total potential energy is a function of the magnitudes $v_{0}$ and $\theta_{0}$. Minimisation of the total potential energy is obtained by equating to 0 its partial derivatives. Expression (15) then rewrites as:

$$
\left\{\begin{array}{l}
\frac{\partial \Pi}{\partial v_{0}} \\
\frac{\partial \Pi}{\partial \theta_{0}}
\end{array}\right\}=\left\{\begin{array}{ll}
a_{11} & a_{12} \\
a_{21} & a_{22}
\end{array}\right\}\left\{\begin{array}{l}
v_{0} \\
\theta_{0}
\end{array}\right\}=\left\{\begin{array}{l}
0 \\
0
\end{array}\right\}
$$

Considering the previous assumptions described in 3.1, the total potential energy is given by:

$$
\Pi=U_{\text {beam }}+U_{\text {spring }}-W
$$

With:

$U_{\text {beam }}=\frac{1}{2} \int\left(E I_{\mathrm{z}} v_{, x x}{ }^{2}+E I_{\mathrm{w}} \theta_{, x x}{ }^{2}+G I_{\mathrm{t}} \theta_{, x}^{2}\right) d x$

$U_{\text {spring }}=c_{\mathrm{w}} \frac{\theta^{\prime}(0)^{2}+\theta^{\prime}(L)^{2}}{2}$

$W=-\int\left(M_{y, 0} \theta v_{, x x}\right) d x$

Where ", $X$ " stands for the first derivative by $\mathrm{x}$ and ",$X X$ ” for the second.

The contribution of the in-plane displacement is generally negligible when compared to that of the out-of-plane displacement and torsional rotation. This contribution is therefore omitted in the expression (17) of the potential energy. Replacing the lateral displacement and the torsional rotation by expressions (8) and (14), the potential energy becomes:

$$
\begin{array}{r}
\Pi=\frac{1}{2}\left(\frac{\pi}{L}\right)^{2}\left[E I_{\mathrm{z}}\left(\frac{\pi}{L}\right)^{2} v_{0}{ }^{2} \frac{L}{2}+E I_{\mathrm{w}} \theta_{0}{ }^{2}\left(\frac{\pi}{L}\right)^{2} \xi_{\mathrm{w}}+G I_{\mathrm{t}} \theta_{0}{ }^{2} \xi_{\mathrm{t}}\right. \\
\left.-2 M_{\mathrm{y}, 0} \theta_{0} v_{0} \sqrt{2 L} \xi_{\mathrm{M}}\right]+\frac{\theta_{0}{ }^{2}}{2}\left(\frac{\pi}{L}\right)^{2} \xi_{\mathrm{s}}
\end{array}
$$

With:

$$
\begin{aligned}
& \xi_{\mathrm{M}}=\pi \sqrt{2 L}+\frac{4 \sqrt{2}}{3} \frac{\sqrt{L}}{\pi} \frac{c_{\mathrm{W}} L}{E I_{\mathrm{w}}} \\
& -- \\
& \xi_{\mathrm{t}}=8 \pi^{2} L+2 L\left(\frac{c_{\mathrm{W}} L}{E I_{\mathrm{w}}}\right)^{2}+\frac{64}{3} L \frac{c_{\mathrm{W}} L}{E I_{\mathrm{w}}} \\
& -- \\
& \xi_{\mathrm{w}}=\xi_{\mathrm{t}}+6 L\left(\frac{c_{\mathrm{W}} L}{E I_{\mathrm{w}}}\right)^{2} \\
& -- \\
& \xi_{\mathrm{s}}=32 c_{\mathrm{w}} \pi^{2}
\end{aligned}
$$

\subsubsection{Bastic critical moment}

Taking the derivative of $\Pi$ with respect to $v_{0}$ and $\theta_{0}$, we obtain two equations:

$$
\begin{gathered}
E I_{\mathrm{z}}\left(\frac{\pi}{L}\right)^{4} v_{0} \frac{L}{2}-M_{\mathrm{y}, 0, \mathrm{cr}} \theta_{0}\left(\frac{\pi}{L}\right)^{2} \xi_{\mathrm{M}} \sqrt{2 L}=0 \\
E I_{\mathrm{w}} \theta_{0}\left(\frac{\pi}{L}\right)^{2} \xi_{\mathrm{w}}+G I_{\mathrm{t}} \theta_{0} \xi_{\mathrm{t}}-M_{\mathrm{y}, 0, \mathrm{cr}} v_{0} \xi_{\mathrm{M}} \sqrt{2 L}+\theta_{0} \xi_{\mathrm{s}}=0
\end{gathered}
$$

From equation (19) arises the relationship between the two magnitudes:

$$
v_{0}=\theta_{0} \frac{M_{\mathrm{y}, 0, \mathrm{cr}}}{E I_{\mathrm{z}}\left(\frac{\pi}{L}\right)^{2}} 2 \sqrt{\frac{2}{L}} \xi_{\mathrm{M}}
$$

The elastic critical bending moment $M_{\mathrm{y}, 0, \mathrm{cr}}$ is then derived from expressions (20) and (21):

$$
M_{\mathrm{y}, 0, \mathrm{cr}}=\sqrt{\frac{\xi_{\mathrm{t}}}{\xi_{\mathrm{M}}^{2}}} E I_{\mathrm{z}}\left(\frac{\pi}{L}\right)^{2} \sqrt{\frac{I_{\mathrm{w}}}{I_{\mathrm{z}}} \frac{\xi_{\mathrm{w}}}{\xi_{\mathrm{t}}}+\frac{\xi_{\mathrm{s}}}{\xi_{\mathrm{t}}} \frac{L^{2}}{E I_{\mathrm{z}} \pi^{2}}+\frac{G I_{\mathrm{t}} L^{2}}{E I_{\mathrm{z}} \pi^{2}}}
$$

From the previous expression, the warping coefficient $k_{\mathrm{w}}$ and the equivalent uniform moment fact or $C_{1}$ are identified as:

$$
k_{\mathrm{w}}=\sqrt{\frac{\pi^{2}+\frac{8}{3} \frac{c_{\mathrm{w}} L}{E I_{\mathrm{w}}}+\left(\frac{1}{2} \frac{c_{\mathrm{W}} L}{E I_{\mathrm{w}}}\right)^{2}}{\pi^{2}+\frac{20}{3} \frac{c_{\mathrm{w}} L}{E I_{\mathrm{w}}}+\left(\frac{c_{\mathrm{w}} L}{E I_{\mathrm{w}}}\right)^{2}}}
$$




$$
C_{1}=\frac{\sqrt{\pi^{2}+\frac{8}{3} \frac{c_{\mathrm{W}} L}{E I_{\mathrm{w}}}+\left(\frac{1}{2} \frac{c_{\mathrm{W}} L}{E I_{\mathrm{w}}}\right)^{2}}}{\pi+\frac{4}{3 \pi} \frac{C_{\mathrm{W}} L}{E I_{\mathrm{w}}}}
$$

The elastic critical bending moment of a doubly symmetric beam under constant moment with warping restraints at supports is given by expression (1) with $k_{w}$ and $C_{1}$ given by (23) and (24).

\subsection{Linear bending moment distribution}

Considering a beam subjected to a linear bending moment distribution with $\psi$ being the ratio between end moments, we derive the expression of the critical bending moment based on the out-of-plane displacement and torsional rotation given by (8) and (14). The following expressions are obtained for coefficients $k_{\mathrm{w}}$ and $C_{1}$ :

$$
\begin{gathered}
C_{1}=\frac{2}{1+\psi} \frac{\sqrt{\pi^{2}+\frac{8}{3} \frac{c_{\mathrm{W}} L}{E I_{\mathrm{w}}}+\left(\frac{1}{2} \frac{c_{\mathrm{W}} L}{E I_{\mathrm{w}}}\right)^{2}}}{\pi+\frac{4}{3 \pi} \frac{c_{\mathrm{w}} L}{E I_{\mathrm{w}}}} \\
k_{w}=\sqrt{\frac{\pi^{2}+\frac{8}{3} \frac{c_{w} L}{E I_{w}}+\left(\frac{1}{2} \frac{c_{w} L}{E I_{w}}\right)^{2}}{\pi^{2}+\frac{20}{3} \frac{c_{w} L}{E I_{w}}+\left(\frac{c_{w} L}{E I_{w}}\right)^{2}}}
\end{gathered}
$$

Expression (26) for $k_{\mathrm{w}}$ is the same as (23) derived under constant bending moment. Therefore, the warping coefficient does not depend on the bending moment distribution. Besides, $C_{1}$ can be expressed as the product of two distinct factors depending on $\psi$ and on the warping stiffness, respectively. The latter is identical to expression (24) of $C_{1}$ obtained for a uniform bending moment.

For free warping at supports, it is clear that expression (25) leads to inconsistencies for negative values of $\psi$. Indeed, when the ratio tends towards $-1, C_{1}$ tends towards the infinity. This discrepancy comes from the displacement and rotation fields used to derive expressions (25) and (26). Equations (8) and (14) are well suited when the bending moment is constant, or if $\psi>0,5$. Therefore, the displacement and rotation fields need to be enriched with a second term for $v$ and $\theta$.

The general expression of the out-of-plane displacement of a beam with free out-of-plane rotation at both supports can be expressed by the following sum:

$$
v(x)=\sum_{i=1}^{\infty} v_{i} \sin \left(i \frac{\pi x}{L}\right)
$$

To avoid a too cumbersome analytical development, we will consider only the first two terms of the series. The out-of-plane displacement is finally given by (29).

A second term is also added to the torsional rotation, fulfilling the kinematical boundary conditions given by (13). The number of sine waves along the beam is increased, giving the following second term:

$$
\theta_{2}(x)=\theta_{2}\left\{\frac{16}{3} \pi \sin \left(3 \frac{\pi x}{L}\right)+\frac{c_{w} L}{E I_{w}}\left[1-\cos \left(\frac{4 \pi x}{L}\right)\right]\right\}
$$

This second term varies between $\sin \left(3 \frac{\pi x}{L}\right)$ when warping is free and $\left[1-\cos \left(\frac{4 \pi x}{L}\right)\right]$ when warping is prevented at both ends. Finally, the new displacement and rotation fields are:

$$
\begin{gathered}
v(x)=v_{1} \sin \left(\frac{\pi x}{L}\right)+v_{2} \sin \left(2 \frac{\pi x}{L}\right) \\
\theta(x)=\theta_{1}\left\{4 \pi \sin \left(\frac{\pi x}{L}\right)+\frac{c_{w} L}{E I_{w}}\left[1-\cos \left(\frac{2 \pi x}{L}\right)\right]\right\} \\
+\theta_{2}\left\{\frac{16}{3} \pi \sin \left(3 \frac{\pi x}{L}\right)+\frac{c_{w} L}{E I_{w}}\left[1-\cos \left(\frac{4 \pi x}{L}\right)\right]\right\}
\end{gathered}
$$

From expressions (29) and (30), the potential energy $\Pi$ of a doubly symmetric beam subjected to end moments with warping restraints at supports is calculated. The critical moment $M_{\mathrm{cr}}$ is then obtained by solving the following equation:

$$
\left[\begin{array}{l}
\frac{\partial \Pi}{\partial v_{1}} \\
\frac{\partial \Pi}{\partial v_{2}} \\
\frac{\partial \Pi}{\partial \theta_{1}} \\
\frac{\partial \Pi}{\partial \theta_{2}}
\end{array}\right]=\left[\begin{array}{cccl}
K_{11} & 0 & M_{\mathrm{cr}} K_{13} & M_{\mathrm{cr}} K_{14} \\
0 & K_{22} & M_{\mathrm{cr}} K_{23} & M_{\mathrm{cr}} K_{24} \\
M_{\mathrm{cr}} K_{13} & M_{\mathrm{cr}} K_{23} & K_{33} & K_{34} \\
M_{\mathrm{cr}} K_{14} & M_{\mathrm{cr}} K_{24} & K_{34} & K_{44}
\end{array}\right]\left[\begin{array}{l}
v_{1} \\
v_{2} \\
\theta_{1} \\
\theta_{2}
\end{array}\right]=\left[\begin{array}{l}
0 \\
0 \\
0 \\
0
\end{array}\right]
$$

With:

$$
\begin{aligned}
& K_{11}=E I_{\mathrm{z}}\left(\frac{\pi}{L}\right)^{4} \frac{L}{2} \\
& K_{22}=8 E I_{\mathrm{z}}\left(\frac{\pi}{L}\right)^{4} L \\
& \text {-- } \\
& K_{33}=G I_{\mathrm{t}}\left(\frac{\pi}{L}\right)^{2} \xi_{\mathrm{t}}+E I_{\mathrm{w}}\left(\frac{\pi}{L}\right)^{4} \xi_{\mathrm{w}}+\left(\frac{\pi}{L}\right)^{2} \xi_{\mathrm{s}} \\
& \text {-- } \\
& K_{44}=G I_{\mathrm{t}}\left(\frac{\pi}{L}\right)^{2} \xi_{\mathrm{t}, 2}+E I_{\mathrm{w}}\left(\frac{\pi}{L}\right)^{4} \xi_{\mathrm{w}, 2}+16\left(\frac{\pi}{L}\right)^{2} \xi_{\mathrm{s}} \\
& K_{13}=-\left(\frac{\pi}{L}\right)^{2} \sqrt{\frac{L}{2}} \xi_{\mathrm{M}}(1+\Psi) \\
& \text {-- } \\
& K_{14}=-\left(\frac{\pi}{L}\right)^{2} \frac{16}{15} \frac{L}{\pi} \frac{c_{\mathrm{w}} L}{E I_{\mathrm{w}}}(1+\Psi) \\
& K_{23}=\left(\frac{\pi}{L}\right)^{2} \frac{L}{\pi} \xi_{\mathrm{M}, 2}(\Psi-1) \\
& \text {-- } \\
& K_{24}=\left(\frac{\pi}{L}\right)^{2} \frac{L}{\pi} \xi_{\mathrm{M}, 3}(\Psi-1) \\
& \text {-- } \\
& K_{34}=-G I_{\mathrm{t}} \frac{\pi^{2}}{L} \frac{c_{\mathrm{W}} L}{E I_{\mathrm{w}}} \frac{256}{15}-E I_{\mathrm{w}}\left(\frac{\pi}{L}\right)^{2} \frac{\pi^{2}}{L} \frac{c_{\mathrm{W}} L}{E I_{\mathrm{w}}} \frac{3328}{15}+4\left(\frac{\pi}{L}\right)^{2} \xi_{\mathrm{s}} \\
& \xi_{\mathrm{t}, 2}=128 \pi^{2} L+\frac{1024}{7} L \frac{c_{\mathrm{w}} L}{E I_{\mathrm{w}}}+8 L\left(\frac{c_{\mathrm{w}} L}{E I_{\mathrm{w}}}\right)^{2} \\
& \xi_{\mathrm{w}, 2}=9 \xi_{\mathrm{t}, 2}+56 L\left(\frac{c_{\mathrm{w}} L}{E I_{\mathrm{w}}}\right)^{2} \\
& \xi_{\mathrm{M}, 2}=\frac{128}{9}+\frac{3}{2} \frac{c_{\mathrm{w}} L}{E I_{\mathrm{w}}} \\
& \xi_{\mathrm{M}, 3}=\frac{512}{25}+\frac{8}{3} \frac{c_{\mathrm{w}} L}{E I_{\mathrm{w}}}
\end{aligned}
$$

The critical bending moment is finally derived by equalling to zero the determinant of the $4 \times 4$ matrix in equation (31). The coefficients $k_{\mathrm{w}}$ and $C_{1}$ are then identified in the $4^{\text {th }}$ degree equation on $M_{\mathrm{cr}}$.

The expression of the warping coefficient $k_{\mathrm{w}}$ is similar to that derived with a constant bending moment (23). The equation on $M_{\text {cr }}$ is 
then simplified as an equation on $C_{1}$. To simplify, we assume that the ratio $\sqrt{E I_{\mathrm{w}} / G I_{\mathrm{t}} L^{2}}$ is null. It leads to the minimum values of $C_{\mathrm{l}}$ and therefore provides conservative values. The equation becomes:

$$
A C_{1}^{4}-B C_{1}^{2}+C=0
$$

With:

$A=\left(\Psi^{2}-1\right)^{2}\left[40856+18612 \frac{c_{\mathrm{W}} L}{E I_{\mathrm{W}}}+3406\left(\frac{c_{\mathrm{W}} L}{E I_{\mathrm{W}}}\right)^{2}+290,1\left(\frac{c_{\mathrm{W}} L}{E I_{\mathrm{W}}}\right)^{3}+\right.$ $\left.9,51\left(\frac{c_{\mathrm{W}} L}{E I_{\mathrm{W}}}\right)^{4}\right]$

$-$

$B=\pi^{2}\left\{8(\Psi+1)^{2}\left\{\frac{\pi^{2}}{2 L^{2}} \xi_{\mathrm{M}}^{2} \xi_{\mathrm{t}, 2}+\left(\frac{16}{15} \frac{c_{\mathrm{w}} L}{E I_{\mathrm{w}}}\right)^{2}\left(\frac{\xi_{\mathrm{t}}}{L}+16 \pi \sqrt{\frac{2}{L}} \xi_{\mathrm{M}}\right)\right\}+\right.$

$\left.\frac{(\Psi-1)^{2}}{2}\left\{\frac{\xi_{\mathrm{M}, 2}{ }^{2} \xi_{\mathrm{t}, 2}+\xi_{\mathrm{M}, 3}{ }^{2} \xi_{\mathrm{t}}}{L}+\frac{512}{15} \frac{c_{\mathrm{W}} L}{E I_{\mathrm{W}}} \xi_{\mathrm{M}, 2} \xi_{\mathrm{M}, 3}\right\}\right\}$

$C=4 \pi^{4}\left\{\frac{\xi_{\mathrm{t}} \xi_{\mathrm{t}, 2}}{L^{2}}-\left(\frac{256}{15} \frac{c_{\mathrm{W}} L}{E I_{\mathrm{W}}}\right)^{2}\right\}$

The positive solution of the previous equation is:

$$
\begin{array}{ll}
-1<\Psi<1 \quad C_{1}=\sqrt{\frac{4 \pi^{2} \xi_{1}}{\left(\Psi^{2}-1\right)^{2}}\left[\Delta-\sqrt{\Delta^{2}-\frac{\left(\Psi^{2}-1\right)^{2}}{4 \pi^{2} \xi_{1} \xi_{3}}}\right]} \\
\Delta=(\Psi+1)^{2}+\xi_{2}(\Psi-1)^{2} \\
\Psi=1 & C_{1}=\frac{1}{\sqrt{8 \xi_{3}}} \\
\Psi=-1 & C_{1}=\frac{1}{\sqrt{8 \xi_{2} \xi_{3}}}
\end{array}
$$

With:

$\xi_{1}=\frac{123058+47499 \frac{c_{\mathrm{w}} L}{E I_{\mathrm{w}}}+7324\left(\frac{c_{\mathrm{w}} L}{E I_{\mathrm{w}}}\right)^{2}+543,4\left(\frac{c_{\mathrm{w}} L}{E I_{\mathrm{w}}}\right)^{3}+16,50\left(\frac{c_{\mathrm{w}} L}{E I_{\mathrm{w}}}\right)^{4}}{40856+18612 \frac{c_{\mathrm{w}} L}{E I_{\mathrm{w}}}+3406\left(\frac{c_{\mathrm{w}} L}{E I_{\mathrm{w}}}\right)^{2}+290,1\left(\frac{c_{\mathrm{w}} L}{E I_{\mathrm{w}}}\right)^{3}+9,51\left(\frac{c_{\mathrm{w}} L}{E I_{\mathrm{w}}}\right)^{4}}$

$\xi_{2}=\frac{18041+6938 \frac{c_{\mathrm{w}} L}{E I_{\mathrm{w}}}+1049\left(\frac{c_{\mathrm{w}} L}{E I_{\mathrm{w}}}\right)^{2}+73,57\left(\frac{c_{\mathrm{w}} L}{E I_{\mathrm{w}}}\right)^{3}+2,01\left(\frac{c_{\mathrm{w}} L}{E I_{\mathrm{w}}}\right)^{4}}{123058+47499 \frac{c_{\mathrm{w}} L}{E I_{\mathrm{w}}}+7324\left(\frac{c_{\mathrm{w}} L}{E I_{\mathrm{w}}}\right)^{2}+543,4\left(\frac{c_{\mathrm{w}} L}{E I_{\mathrm{w}}}\right)^{3}+16,50\left(\frac{c_{\mathrm{w}} L}{E I_{\mathrm{w}}}\right)^{4}}$

$\xi_{3}=\frac{123058+47499 \frac{c_{\mathrm{w}} L}{E I_{\mathrm{w}}}+7324\left(\frac{c_{\mathrm{w}} L}{E I_{\mathrm{w}}}\right)^{2}+543,4\left(\frac{c_{\mathrm{w}} L}{E I_{\mathrm{w}}}\right)^{3}+16,50\left(\frac{c_{\mathrm{w}} L}{E I_{\mathrm{w}}}\right)^{4}}{984463+379988 \frac{c_{\mathrm{w}} L}{E I_{\mathrm{w}}}+59097\left(\frac{c_{\mathrm{w}} L}{E I_{\mathrm{w}}}\right)^{2}+4572\left(\frac{c_{\mathrm{w}} L}{E I_{\mathrm{w}}}\right)^{3}+157,9\left(\frac{c_{\mathrm{w}} L}{E I_{\mathrm{w}}}\right)^{4}}$

$C_{1}$ depends on the ratio $\psi$ and on the warping stiffness through the parameters $\xi_{\text {i. }}$ In the specific cases of free and fixed warping at both supports, the values of $\xi_{1}, \xi_{2}$ and $\xi_{3}$ are given in Table 3 .

Table 3 Values of $\xi_{1}, \xi_{2}$ and $\xi_{3}$ when free or fixed warping

\begin{tabular}{cccc}
\hline & $\xi_{1}$ & $\xi_{2}$ & $\xi_{3}$ \\
\hline $\begin{array}{c}\text { Free warping } \\
\left(\boldsymbol{c}_{\mathbf{w}}=\mathbf{0}\right)\end{array}$ & 3,012 & 0,147 & 0,125 \\
$\begin{array}{c}\text { Fixed warping } \\
\left(\boldsymbol{c}_{\mathbf{w}} \rightarrow \infty\right)\end{array}$ & 1,735 & 0,122 & 0,104 \\
\hline
\end{tabular}

The values of $C_{1}$ are calculated in Table 4 for various values of $\psi$ in the two extreme cases of free and fixed warping at supports. We can notice an increase in the values of $C_{1}$ from 9,4 to almost $20 \%$ when warping is fixed.

Values of $C_{1}$ given by TableM.1 from the French National Annex to Eurocode 3 Part 1-1 [2] are also presented in Table 4 for free warping. The analytical model give results close to the French NA even though small differences, below $15 \%$, can be noticed. Again, like in

\begin{tabular}{|c|c|c|c|c|}
\hline \multirow[b]{2}{*}{$\psi$} & \multicolumn{2}{|c|}{$C_{1}$} & \multirow[b]{2}{*}{$\begin{array}{c}C_{1, \text { fixed warp. }} / \\
C_{1, \text { freewarp. }}\end{array}$} & \multirow[b]{2}{*}{$\begin{array}{c}C_{1} \text { French NA } \\
\text { [2] }\end{array}$} \\
\hline & $\begin{array}{c}\text { Free } \\
\text { warping }\end{array}$ & $\begin{array}{c}\text { Fixed } \\
\text { warping }\end{array}$ & & \\
\hline 1 & 1,00 & 1,09 & 1,094 & 1,00 \\
\hline 0,75 & 1,14 & 1,25 & 1,094 & 1,14 \\
\hline 0,5 & 1,32 & 1,45 & 1,096 & 1,31 \\
\hline 0,25 & 1,56 & 1,72 & 1,102 & 1,52 \\
\hline 0 & 1,88 & 2,10 & 1,114 & 1,77 \\
\hline$-0,25$ & 2,28 & 2,60 & 1,141 & 2,05 \\
\hline$-0,5$ & 2,67 & 3,14 & 1,179 & 2,33 \\
\hline$-0,75$ & 2,81 & 3,37 & 1,198 & 2,57 \\
\hline-1 & 2,61 & 3,13 & 1,198 & 2,55 \\
\hline
\end{tabular}
Table 2 where Piotrowski's [6] resultswere found to be greater than the French NA when warping isfree, finite element analyses must be computed in order to assess the safety level of the two approaches.

Table $4 G$ for free or fixed warping at supports

In Figure 5 , the warping coefficient $k_{w}$ is plotted against the parameter $\frac{c_{\mathrm{w}} L}{E I_{\mathrm{W}}}$ accounting for the warping restraints. It shows the results obtained with the proposals of Lindner and Gietzelt[3] and ECCS[4], Pi and Trahair [5], Piotrowski and Szychowski [6] and expression (23).

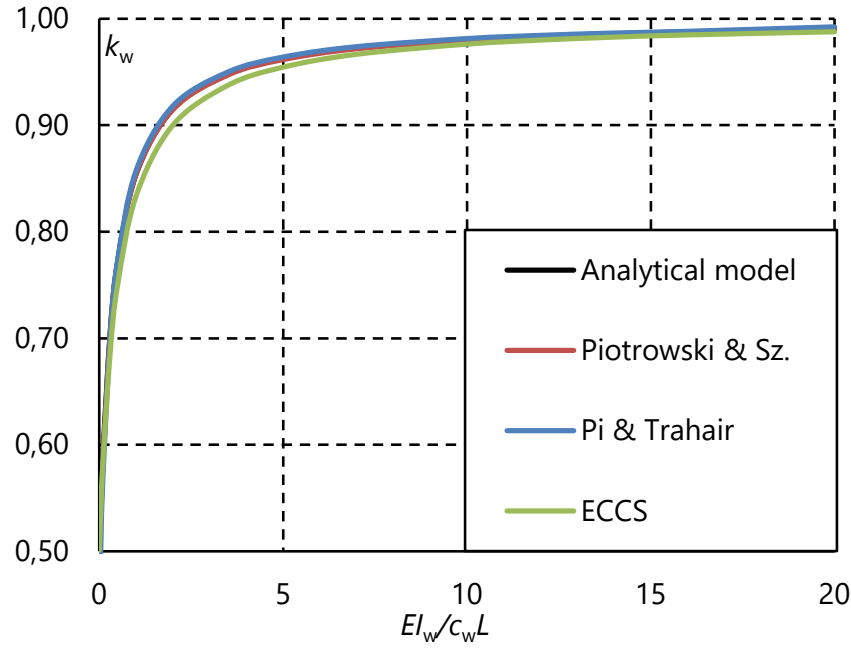

Figure 5 Warping coefficient $k_{w}$ against $\frac{E I_{\mathrm{w}}}{c_{\mathrm{w}} L}$

The different proposals give close results. Some differences appear when the restraints tend to fix warping, i.e. when $\boldsymbol{c}_{\mathrm{w}} \rightarrow \infty$. Indeed, $\mathrm{Pi}$ 
and Trahair tend towards 0,44 while the other proposals tend towards 0,5 .

From the analytical model developed in $\S 3$, expressions of the equivalent uniform moment factor $C_{1}$ and the warping coefficient $k_{w}$ were derived to determine the elastic critical bending moment of a uniform beam with doubly symmetric I-section subjected to linear bending moment distribution with warping restraints at supports. What ever the bending moment distribution, $k_{w}$ only depends on the warping stiffness, as expressed in equation (23). However, $C_{1}$ depends on both the warping stiffness and the bending moment distribution as expressions (33) to (35) state. Formulae derived from the analytical model will be compar ed against finite element analyses in $\S 4$.

\section{$4 \quad$ Finite Eement Analyses}

\subsection{Numerical Models}

Two finite element models are used for comparisons:

- $\quad$ Shell elements with ANSYS

- $\quad$ Beam elements with LTBeamN [1].

Linear Bifurcation Analyses (LBA) are performed considering Young's modulus $E=210000 \mathrm{MPa}$ and Poisson's ratio $v=0,3$.

The geometry of the studied beams is given in Table 5. Warping is restrained by end plates, fixed or free at both ends. Thickness of the end plates varies between 7 and $70 \mathrm{~mm}$ to extend the study beyond the common practice.

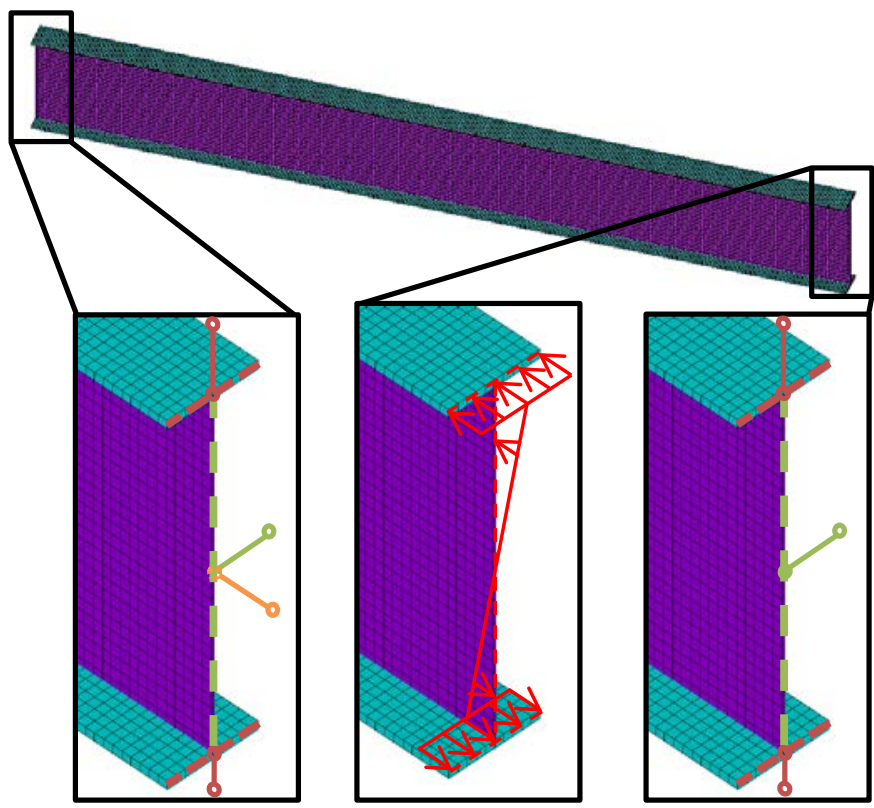

Figure 6 End moment and fork support conditions

The shell element model is developed using 8-node elements, with 6 degrees of freedom at each node. The out-of-plane displacement and torsional rotation of the section are fixed at supports as well as the axial displacement of web centre at one end to reproduce typical fork support conditions (see Figure 6). End plates are modelled at supports with shell elements. In addition, distortion is prevented along the beam.

Eventually, nodal forces along the $x$-axis are applied to provoke end moments, leading to a linear bending moment distribution as shown in Figure 6.
Figure 7 exhibits the out-of-plane displacements corresponding to the critical mode of beam P2 subjected to a) uniform and b) linear bending moment distribution with $\psi=-1$, along with $20 \mathrm{~mm}$-thick end plates. The two modal shapes are quite different. Under constant bending moment, half of a sine wave is visible while a complete sine-wave appear for $\psi=-1$. These observations are consistent with the displacement fields assumed in §3.3.
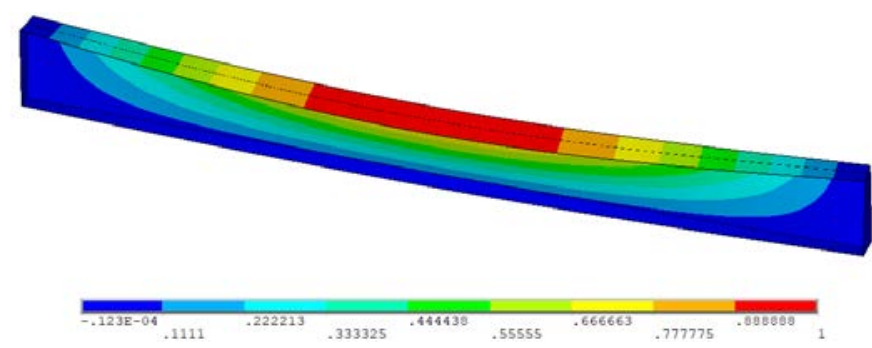

a) $\psi=1$

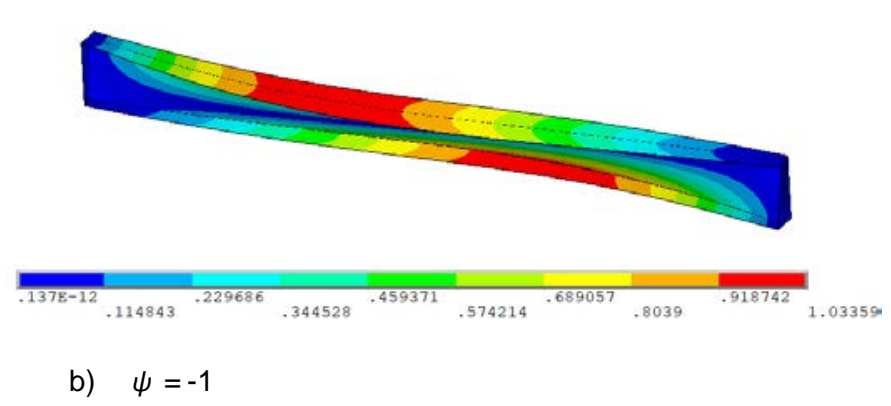

Figure 7 Out-of-plane displacement of P2 with $20 \mathrm{~mm}$-thick end plates

The beam element model is developed with two-noded beam elements with 7 degrees of freedom (including warping) at each node. Fork support conditions are enforced by preventing lateral displacement and torsional rotation at both ends and longitudinal displacement at one end.

The degree of freedom corresponding to warping may be fixed or connected to a warping spring with a stiffness $c_{w}$, calculated according to equation (3). End moments are directly applied to induce a linear bending moment distribution.

Table 5 Geometry of studied beams

\begin{tabular}{cccccc}
\hline Beam & $\mathrm{h}_{\mathrm{s}}(\mathrm{mm})$ & $\mathrm{t}_{\mathrm{w}}(\mathrm{mm})$ & $\mathrm{b}(\mathrm{mm})$ & $\mathrm{t}_{\mathrm{f}}(\mathrm{mm})$ & $\mathrm{L}(\mathrm{m})$ \\
\hline P1 & 600 & 6 & 200 & 14 & 6 \\
P2 & 800 & 10 & 280 & 14 & 10 \\
P3 & 800 & 6 & 280 & 12 & 10 \\
P4 & 300 & 4 & 100 & 6 & $6 \& 9$ \\
P5 & 300 & 6 & 100 & 10 & $6 \& 9$ \\
P6 & 347,3 & 8 & 170 & 12,7 & 6 \\
P7 & 337,5 & 12,5 & 300 & 22,5 & $3 \& 6$ \\
\hline
\end{tabular}

\subsection{Comparison with the Analytical Model}

\subsubsection{Equivalent uniform moment factor $\mathrm{C}_{1}$}

Finite element analyses have been performed with the beam model using LTBeamN and compared against analytical predictions. The 
coefficient $C$ was determined for a beam under linear bending moment distribution in the cases of (i) free warping and (ii) fixed warping at both ends and compared against both analytical model and the French National Annex [2].

Analyses were performed on beams P1, P2, P3, P6 and P7 (see Table 5). Figure 8 presents the values of $C_{1}$ as a function of $\psi$.

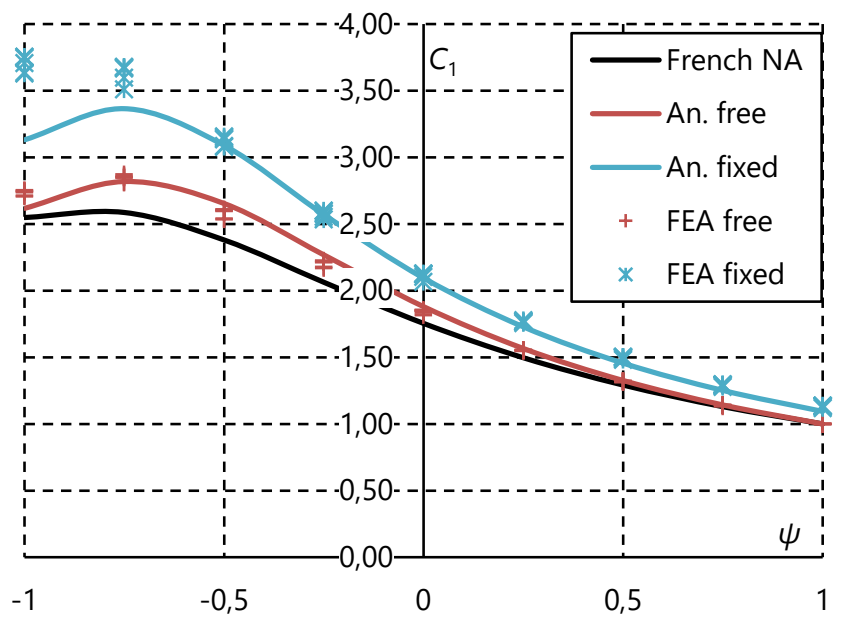

Figure $8 C_{1}$ for free or fixed warping

In addition to the values given in the French National Annex [2], the figure shows the results obtained with finite element analyses (FEA) and the analytical model, i.e. expressions (33) to (35), in the cases of free warping (referred to as "free") and fixed warping (referred to as "fixed").

For free warping, the analytical model is in good agreement with FEA results. The value of $C_{1}$ might be slightly overestimated (of less than $3 \%$ ) when $\psi$ is between $-0,5$ and 0 but the French NA is quite conservative : $C_{1}$ may be underestimated up to $10 \%$.

For fixed warping, Figure 8 shows a good agreement between the numerical and analytical modelswhen $\psi$ is greater than $-0,75$. When the ratio is below that value, the analytical model lays on the safe side, 15\% lower than FEA results. The deviation between the values of $C_{1}$ given by the French NA and FEA results when warping is fixed is up to $45 \%$, confirming that the French NA is overly conservative when warping is fixed.

\subsubsection{Bastic critical bending moment}

To state about the consistency of the complete analytical model, analyses were conducted using the two finite element models. The values of the elastic critical bending moment $M_{\text {cr }}$ resulting from FE analyses were then compared against predictions of the analytical model as well as Piotrowski's [6] proposals.

The ratio $M_{\mathrm{cr}} / M_{\mathrm{cr}, 0}$, with $M_{\mathrm{cr}, 0}$ being the critical bending moment when warping is free, is plotted against the end plates'thickness for beam P2 in Figure 9a) to c). Thickness $t_{p}$ of the end plates is directly connected to the warping stiffness $c_{\mathrm{w}}$ given by expression (3).

A jump in the values of $t_{\mathrm{p}}$ can be seen on Figure 9a) to c), between a $70 \mathrm{~mm}$ thickness and infinite thickness corresponding to fixed warping conditions. The analytical model depicted in this article and formula (5) from Piotrowski [6] are represented by continuous curves for thicknesses ranging between 0 and $70 \mathrm{~mm}$ and by empty triangles for fixed warping.

Figure 9 shows a significant increase of the critical bending moment when warping is fixed. In every cases, its value is at least almost doubled ( $+95 \%$ or more)

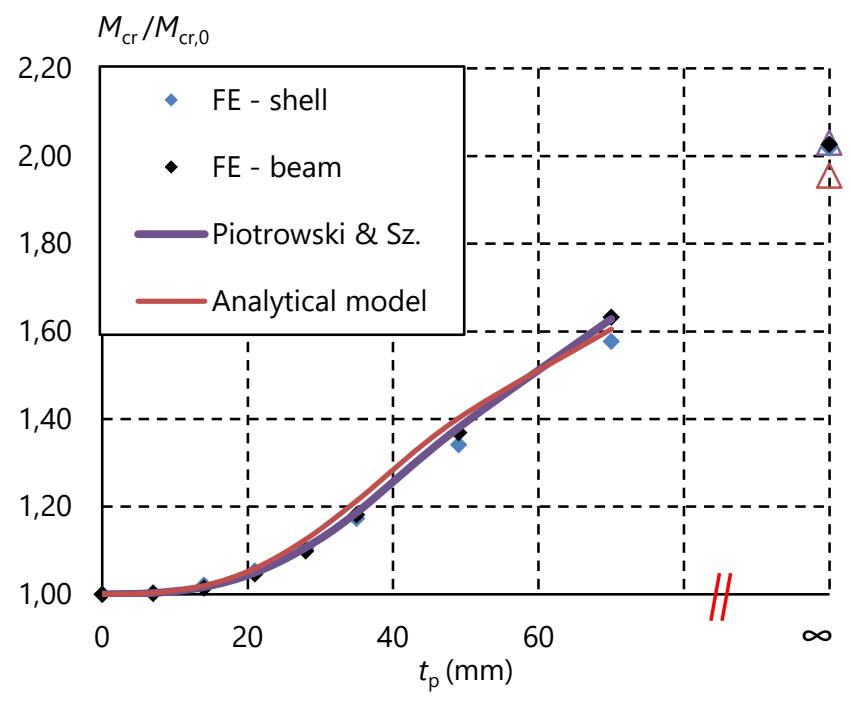

a) $\quad \psi=1$

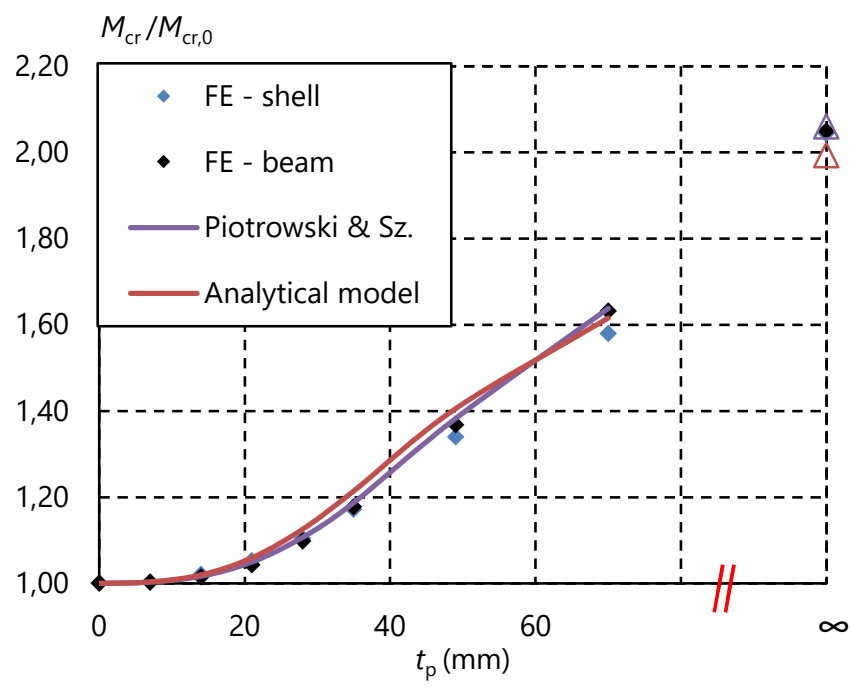

b) $\quad \psi=0$

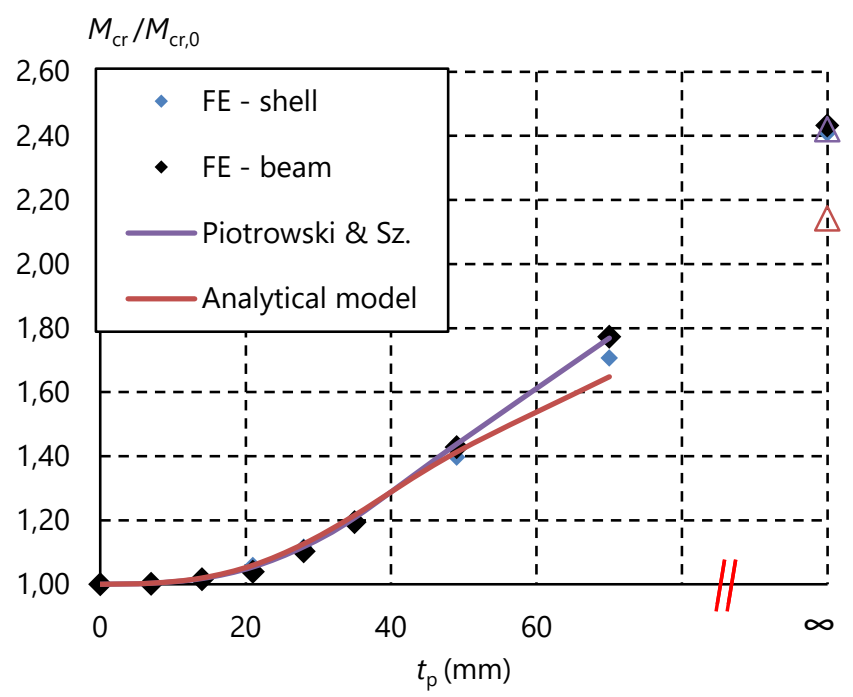

c) $\quad \psi=-1$

Figure 9 Increase in $M_{\mathrm{cr}}$ for $\mathrm{P} 2$ under linear moment with warping restraints

When the bending moment distribution is uniform or triangular, all the methods give the same results up to $30 \mathrm{~mm}$-thick end plates. 
When the warping stiffness increases, the analytical model hardly overestimates (of no more than 5\%) the value of the elastic critical bending moment when compared to finite element analyses. When warping is fixed, the analytical model becomes slightly safer, with a difference below $3 \%$.

When $\psi=-1$, the results given by the different methods coincide up to a higher warping restraint. When the warping stiffness increases, the analytical model lays on the safe side but the difference with the shell model is not greater than $10 \%$.

The variation between Piotrowski [6] and the analytical model is only visible when the warping restraints are significant, particularly when $\psi=-1$. When compared to finite element analyses using shell elements, the analytical model always lays on the safe side unlike $\mathrm{Pi}$ otrowski [6].

Table 6 shows statistical parameters about the calculated values of the critical moment of the beams P1 to P5 (included) presented in Table 5. The ratios between critical bending moments determined with the analytical model and the shell finite element model are presented in Table 6 . This table also shows the relations between the critical bending moment increases $M_{\mathrm{cr}} / M_{\mathrm{cr}, 0}$ calculated with the analytical model and FE analyses.

Table 6 Comparison between analytical results and shell finite element analyses

\begin{tabular}{|c|c|c|c|c|c|c|}
\hline$\psi$ & & 1 & 0,5 & 0 & $-0,5$ & -1 \\
\hline$\left(\frac{M_{\mathrm{cr}}}{M_{\mathrm{cr}, 0}}\right)_{\text {an }}$ & $M$ & 1,006 & 1,006 & 1,007 & 1,005 & 0,976 \\
\hline$\left(\frac{M_{\mathrm{cr}}}{M_{\mathrm{cr}, 0}}\right)_{\mathrm{FEA}}$ & SD & 0,0201 & 0,0201 & 0,0206 & 0,0178 & 0,0352 \\
\hline$M_{\mathrm{cr}, \mathrm{an}}$ & $M$ & 1,017 & 1,021 & 1,043 & 1,069 & 0,954 \\
\hline$\overline{M_{\text {cr,FEA }}}$ & SD. & 0,0207 & 0,0206 & 0,0220 & 0,0275 & 0,0345 \\
\hline
\end{tabular}

Where:

- M:Mean

- SD: Standard deviation

The elastic critical bending moment evolution estimated by the analytical model is very close to the results of shell finite element analyses. The difference is lower than $1 \%$ except when $\psi=-1$ where it increases up to $2,4 \%$.

Critical bending moments determined with the analytical model and FEA are slightly different, but their difference is less than 7\%, particularly when $\psi$ is between $-0,5$ and 0 . It matches with the values of $\psi$ for which the analytical and numerical models show small differences in the value of $C_{1}$ for free warping (see Figure 8).

The displacement and rotation fields could still be refined with a third term to improve results. However, it would lead to heavier analytical developments, leading to a very cumbersome expression for the equivalent uniform moment factor $C_{1}$. In addition, $7 \%$ is still an admissible difference given that the resistant bending moment determined according to Eurocode 3 Part 1-1 [7], $M_{b, R d}$, depends not only on the critical bending moment but also on many other parameters.

\section{$5 \quad$ Conclusions}

The energy method has been used to derive an analytical expression of the elastic critical bending moment of a beam with uniform doubly symmetric I-section and warping restraints at both ends, subjected to a uniform or linear bending moment distribution. The analytical expression is the same as the French National Annex to Eurocode 3 Part 1-1 [2], but the warping coefficient $k_{\mathrm{w}}$ and the equivalent uniform moment factor $C_{1}$ have distinct expressions.

The warping coefficient $k_{\mathrm{w}}$ has been shown to remain unchanged whatever the bending moment distribution and only depends upon the warping stiffness of the beam $E_{\mathrm{w}}$, its length $L$ and the stiffness of the warping restraints $c_{\mathrm{w}}$. Furthermore, the equivalent uniform moment factor $C_{1}$, only based on the bending moment distribution in the French NA [2], is found to also be depending on the stiffness $c_{\mathrm{w}}$ of the warping restraints.

Results from the analytical model were shown to be in good agreement with finite element analyses computed with the software packages ANSYS and LTBeamN [1].

The increase in the value of the critical moment is limited if the warping restraints are only obtained by end plates, but the benefit can become significant if different end connections are considered (fixed column bases, beam-to-beam or beam-to-column joints..).

Future work may include the derivation of analytical expressions to determine the warping stiffness corresponding to realistic support conditions. In addition, the bending moment distribution may be extended to generalise the expression of the elastic critical moment.

\section{References}

[1] CTICM. Software LTBeamN, v1.0.3, available on < https://www.cticm.com/logiciel/ltbeamn/ >.

[2] AFNOR (2013) NFEN1993-1-1/NA : Eurocode3-Design of steel structures-Part 1-1: General rulesand rulesfor buildings-French National Annex to EN 1993-1-1.

[3] Lindner, J.; Gietzelt, R. (1984) Stabilisierung von biegeträgern mit I-Profil durch angeschweißte kopfplatten. Stahlbau, vol. 3, pp 6974.

[4] ECCS, Technical Committee 8 - Stability (2006) Publication 119: Rules for member stability in EN 1993-1-1 : background documentation and design guidelines.

[5] Pi, Y.; Trahair, N. (2000) Distortion and warping at beam supports Journal of Structural Engineering, vol. 126, pp 1279-87.

[6] Piotrowski, R.; Szychowski, A. (2019) Lateral-torsional buckling of steel beams elastically restrained at the support nodes. Applied Sciences, vol. 9 (9), 1944.

[7] CEN (2005) EN 1993-1-1 : Eurocode 3-Design of steel structures - Part 1-1: General rules and rules for buildings. European Committee for Standardisation, Brussels.

[8] Vlasov, V.(1961) Thin-walled elastic beams(2 $2^{\text {nd }}$ edition).

[9] Djalaly, H. (1974) Calcul de la résistance ultime au déversement. Revue Construction Métallique $n^{\circ} 1$,pp 58-77.

[10] Timoshenko, S.; Gere, J. (1963) Theory of elastic stability. McGraw-Hill Book Company 